University of Wollongong

Research Online

Faculty of Engineering and Information

Faculty of Engineering and Information

Sciences - Papers: Part A

Sciences

$1-1-2014$

\title{
Variational inequality model for cordon-based congestion pricing under side constrained stochastic user equilibrium conditions
}

Zhiyuan Liu

Monash University

Qiang Meng

National University of Singapore

Shuaian Wang

University of Wollongong, shuaian@uow.edu.au

Follow this and additional works at: https://ro.uow.edu.au/eispapers

Part of the Engineering Commons, and the Science and Technology Studies Commons

Research Online is the open access institutional repository for the University of Wollongong. For further information contact the UOW Library: research-pubs@uow.edu.au 


\title{
Variational inequality model for cordon-based congestion pricing under side constrained stochastic user equilibrium conditions
}

\begin{abstract}
A major objective of the practical implemented cordon-based congestion pricing schemes is to maintain the traffic conditions within the cordon area, which is rarely considered in most of the existing studies. Thus, this paper addresses the optimal toll charge pattern that can restrict the total inbound flow of each cordon to a predetermined threshold. The toll charges on all the entry links of one cordon are required to be identical, for the ease of implementation and users' recognition. The users' route choice behaviour is assumed to follow stochastic user equilibrium (SUE) with asymmetric link travel time functions. It is shown that such an optimal toll charge pattern can be attained by solving a SUE problem with side constraints.A variational inequality $(\mathrm{VI})$ model is first proposed for the optimal toll pattern, where the monotone property of this model is rigorously proved. Then, a convergent self-adaptive prediction and correction method can be adopted for solving the VI model. It is shown that when used in practice, the solution method only needs traffic counts on entry links of each cordon.
\end{abstract}

\section{Keywords}

inequality, model, variational, cordon, conditions, congestion, pricing, under, side, constrained, stochastic, user, equilibrium

\section{Disciplines \\ Engineering | Science and Technology Studies}

\section{Publication Details}

Liu, Z., Meng, Q. \& Wang, S. (2014). Variational inequality model for cordon-based congestion pricing under side constrained stochastic user equilibrium conditions. Transportmetrica A: Transport Science, 10 (8), 693-704. 


\title{
VARIATIONAL INEQUALITY MODEL FOR CORDON-BASED CONGESTION PRICING UNDER SIDE CONSTRAINED STOCHASTIC USER EQUILIBRIUM CONDITIONS
}

\author{
Zhiyuan Liu*a\&b, Qiang Meng ${ }^{b}$, Shuaian Wang ${ }^{c}$ \\ ${ }^{a}$ Institute of Transport Studies, Department of Civil Engineering, Monash University, \\ Clayton, Victoria 3800, Australia \\ ${ }^{b}$ Department of Civil and Environmental Engineering, National University of Singapore, \\ Singapore 117576, Singapore \\ ${ }^{c}$ School of Mathematics and Applied Statistics, University of Wollongong, Wollongong, \\ NSW 2522, Australia
}

\begin{abstract}
A major objective of the practical implemented cordon-based congestion pricing schemes is to maintain the traffic conditions within the cordon area, which is rarely considered in most of the existing studies. Thus, this paper addresses the optimal toll charge pattern that can restrict the total inbound flow of each cordon to a predetermined threshold. The toll charges on all the entry links of one cordon are required to be identical, for the ease of implementation and users' recognition. The users' route choice behavior is assumed to follow stochastic user equilibrium (SUE) with asymmetric link travel time functions. It is shown that such an optimal toll charge pattern can be attained by solving a SUE problem with side constraints. A variational inequality (VI) model is first proposed for the optimal toll pattern, where the monotone property of this model is rigorously proven. Then, a convergent self-adaptive prediction and correction method can be adopted for solving the VI model. It is shown that when used in practice, the solution method only needs traffic counts on entry links of each cordon.
\end{abstract}

Key words: cordon-based pricing; side constraint; variational inequality; stochastic user equilibrium

\footnotetext{
* Corresponding author

Tel.: +61-3-99054951

Fax: +61-3-99054944

E-mail address: zhiyuan.liu@monash.edu (Z. Liu); ceemq@ nus.edu.sg (Q. Meng);

wangshuaian@gmail.com (S. Wang)
} 


\section{INTRODUCTION}

\subsection{Background and Objectives}

Traffic congestions impose a great burden on the smooth operation of urban cities. Congestion pricing is well recognized to be an effective instrument to alleviate the traffic congestions (see, Yang and Huang, 1998; Lawphongpanich et al. 2006; de Palma and Lindsey, 2011, among many others). Ever since Pigou (1920), a long history of nearly one hundred years can be observed for the theoretical studies of congestion pricing with fruitful achievements. Most of these studies focus on first-best or second-best pricing with the aim of optimizing one or more index of the entire transport system. These indexes include total travel time, total social benefit and total toll revenue.

However, the theoretical achievements, like first- and second- best pricing, have rarely been adopted in practical implementations. This is mainly because of two reasons: (a) most of the theoretical studies highly rely on the data of each network attribute. These data include origin-destination (OD) demand, link travel time functions and network users' value-of-time, etc. The accuracy level of these data would inherently influence the function of each theoretical method, yet it is extremely difficult to obtain these data in practice; (b) another reason is that instead of optimizing a systematic index, the major concern of transport authorities is usually to maintain traffic conditions in CBD area, since the CBD area has high travel demand and traffic congestions in CBD would have larger burden on the economic development. Thus, due to these reasons, most of the practical congestion pricing schemes in urban cities are area-based (e.g., the Area Licensing Scheme in Singapore from 1975 to 1997 and the London Congestion Charging Scheme ever since 2003) or cordon-based (the Electronic Road Pricing system in Singapore and the Stockholm Congestion Charge). These two practical schemes first define one or more restricted zone, usually in the CBD area, and any vehicle accessing any restricted zone from external area must buy a permission license (area-based scheme) or pay a toll at each entry link of this zone (cordon-based scheme). By restricting the total inbound flows, the traffic congestions in the restricted zones are largely mitigated; for instance, shortly after the implementation of Area Licensing Scheme in Singapore after 
1975, the rush-hour inbound traffic to the restricted zone has been reduced by $45 \%$ (Foo, 2000).

Hence, this paper addresses the toll setting problem of cordon-based pricing, with the objective of limiting the total inbound flow to the restricted zone rather than optimizing a systematic index. A threshold should be predefined by the government, and the optimal toll pattern should restrict the total inbound flow below the threshold. Considering the equity issue and in order to avoid over-charge, the toll charge to one cordon should be zero if the total inbound flow is strictly less than its threshold.

In view of the two practices of cordon-based pricing (in Singapore and Stockholm), an interesting feature can be observed: the toll charges on all the entries of one pricing cordon (restricted zone) are identical; for example, identical toll charges are required on the entry links to the Orchard Cordon and Bugis-Marina Centre of Electronic Road Pricing (ERP) system in Singapore. The identical toll charges for one cordon are easier for the network authorities to distribute the toll fares and also convenient for the users' recognition. However, this feature is not considered in previous theoretical studies for cordon-based pricing, thus it is taken into consideration in this paper.

To sum up, the optimal toll pattern addressed in this paper is assumed to fulfill two conditions: (a) total inbound flow of each pricing cordon cannot exceed the threshold; (b) the toll charges on all the entry links to one cordon must be identical. The optimal toll pattern reflects some congestion pricing schemes implemented in practice; for instance, Singapore's Electronic Road Pricing system uses travel speed in the pricing cordon as a key performance index to periodically adjust the toll charges (Liu et al., 2013). These characteristics of practical congestion pricing schemes should also be considered; namely OD demand information, link travel time functions and network users' value-of-time should be obviated for determining the optimal toll pattern. Thus, we intend to propose a solution method that only relies on the traffic count data on the entry links of a pricing cordon, which are convenient to be accurately collected.

Theoretical studies of toll setting problem highly rely on the traffic assignment problem, since the impact of any toll pattern on the network flows should be analyzed by solving a traffic assignment problem. To better reflect the practical conditions, the stochastic user equilibrium (SUE) with asymmetric link travel time functions (see, Sheffi 
and Daganzo, 1977; Liu, 2011) is adopted as a framework for the traffic assignment problem. It should be pointed out that, in practice, the equilibrium link flows on each link can be easily obtained through traffic survey.

We intend to formulate this toll setting problem as a SUE problem with asymmetric link travel time functions and side constraints. It can be shown that the optimal Lagrangian multipliers to the side constraints can fulfill the conditions for optimal toll pattern. Yet, due to the complexity of SUE, formulation for the SUE problem with side constraints is a highly challenging topic. Thus, in spite of its theoretical significance, the general SUE problem with side constraints is still an open question. Hence, we aim to propose a mathematical model, which can successfully formulate this problem and also guarantee the global convergence of a solution algorithm to solve the optimal toll.

The contributions of this paper, in general, are twofold: first, a methodology is proposed for the addressed practical cordon-based pricing scheme, with the objective of maintaining traffic conditions within each cordon area, and moreover the toll charge on each entry of one cordon is required to be identical; second, a mathematical model and convergent algorithm are proposed for the SUE problem with asymmetric link travel time functions and side constraints, which also contribute to the theoretical studies of traffic assignment.

\subsection{Literature Review}

Based on given cordon locations, the toll setting problem for cordon-based pricing is usually taken as a special case of second-best pricing, which is usually formulated as a bi-level model or mathematical program with equilibrium constraints (MPEC) (see, Yang and Zhang, 2002; Verhoef, 2002; Mun et al., 2003; Zhang and Yang, 2004; Maruyama and Sumalee, 2007; Meng and Liu, 2012a; Liu and Meng, 2012; Meng et al., 2012; to name a few). However, the previous studies have seldom taken maintaining traffic conditions in CBD area as an objective. Thus, compared with the no-toll case, the optimal second-best pricing may even give rise to a worse traffic condition in the cordon area.

As discussed above, for the optimal toll pattern addressed in this paper, which can reflect the two features of practical cordon-based pricing schemes, it should be 
formulated as a SUE problem with side constraints. In the literature, the deterministic user equilibrium (DUE) problem with side constraints has been well studied with numerous achievements: Larsson and Patriksson (1999) illustrated that by adding the side constraints to the standard DUE model can obtain a suitable model, which can be solved by a Lagrangian dual method. Nagurney and Ramanujam (1998) proposed an optimization model for the side constrained DUE with multi travel modes, in order to limit the total emission of the transport system. Shahpar et al. (2008) further proposed a framework for solving the dynamic user equilibrium problem with side constraints. A recent study conducted by Chen et al. (2011) has discussed different function types for the physical and environmental constraints of the network, which are taken as side constraints for the DUE model. A VI model was proposed by Chen et al. (2011) to formulate the DUE problem with side constraints. Taking as a simple case of the side constraints, the link capacity constraints problem in the context of DUE has also been widely investigated, see, e.g., Larsson and Patriksson (1995), Nie et al. (2004).

However, due to the complexity of SUE, modeling for the SUE problems with side constraints is more difficult, because directly adding side constraints to the standard SUE model is not effective, see Section 2.3 below for details. Thus, theoretical studies for SUE problem with side constraints or link capacity constraints are quite limited. Bell (1995) solved the logit-based SUE problem with link capacity constraints based on the theory of entropy maximization. However, the achievements in Bell (1995) are not available for the case of general SUE or probit-based SUE. For general SUE with side constraints, Meng et al. (2008) provided a convex minimization model, which is solved by the Lagrangian dual method. The work of Meng et al. (2008) was later extended by Meng and Liu (2011) to the elastic demand case, and further extended to consider the asymmetric link travel times in Meng et al. (2013). However, these studies only consider link capacity constraints. Thus, formulation for the SUE problem with side constraints and asymmetric link travel time functions is still an open question, which is addressed in this paper. In this paper, we first mathematically describe the addressed problem, and then show the challenges of modeling for side constrained SUE problem. Then, a variational inequality model is proposed, whose monotone property is rigorously proven. The monotone property assures the convergence of a prediction and correction method 
with self-adaptive step sizes to solve the VI model. It should be noted that, when used in real world, the solution method only requires the data of traffic counts on the entry links, while obviates the data of OD demand, link travel time functions, as well as drivers' value-of-time.

\section{PROBLEM STATEMENT}

\subsection{Notations and Assumptions}

Consider a strongly connected transport network $G=(N, A)$, where $N$ denotes the set of nodes and $A$ is the set of links. Let $v_{a}$ be the flow on link $a \in A$. The origindestination (OD) pairs on the network are grouped in set $W$, and for each OD pair $w \in W$, its travel demand is $q_{w}$. It is assumed in this paper that $q_{w}$ is constant and fixed. There is at least one path connecting each OD pair $w \in W$, and all the paths between OD pair $w \in W$ are enclosed in set $R_{w}$. Let $f_{w k}$ denote the flow on path $k \in R_{w}$, and the following conservation equations should be fulfilled:

$$
\begin{gathered}
q_{w}=\sum_{k \in R_{w}} f_{w k} \delta_{k}^{w}, \\
v_{a}=\sum_{w \in W} \sum_{k \in R_{w}} f_{w k} \delta_{a k}^{w}, \\
f_{w k} \geq 0,
\end{gathered}
$$

where $\delta_{k}^{w}$ is the OD-path incidence relationship, namely $\delta_{k}^{w}=1$ if path $k$ connects OD pair $w \in W$, and $\delta_{k}^{w}=0$, otherwise. Moreover, $\delta_{a k}^{w}$ is the path-link incidence relationship: $\delta_{a k}^{w}=1$ if link $a$ is on path $k \in R_{w}$ and $\delta_{a k}^{w}=0$, otherwise. Any flow pattern that can satisfy eqs. (1)-(3) is regarded as feasible. Let $\mathbf{v}, \mathbf{f}$ and $\mathbf{q}$ be the column vector for link flows, path flows and OD demands, respectively. Travel time on link $a \in A$ is denoted by $t_{a}$. This paper assumes the asymmetric link travel times, i.e., $t_{a}=t_{a}(\mathbf{v})$ is a function of the link flow vector $\mathbf{v}$. 
Assumption 1. The column vector of link travel time functions $\mathbf{t}(\mathbf{v})=\left(t_{a}(\mathbf{v}), a \in A\right)^{\mathrm{T}}$ is assumed to be strictly monotone and continuously differentiable, which is termed as asymmetric link travel time functions in the literature.

It should be noted that the separable link travel time functions assumed in standard traffic assignment problems, where link travel time is only affected by its own flows, is a special case of the asymmetric link travel time functions. In practice, the travel times on some links, however, are largely influenced by flows on other links; for example, on a two way road with no median barriers separating the flows of traffic, and on an unsignalized network with both arterial and minor roads where the minor roads should give way to arterial roads (Liu, 2011). Assumption 1 hence makes the proposed model in this paper better reflect the practical conditions.

Suppose the total number of cordons on the network is $I$. Each cordon guards a particular area, and each entry to this area is charging. All the entry links to cordon $i \leq I$ are grouped into set $\bar{A}_{i}$, where $\bar{A}_{i} \subseteq A$. The entry links $\bar{A}_{i}, i=1,2, \ldots, I$ of different cordons are mutually exclusive. For the cordon-based pricing, the toll charges on each link in set $\bar{A}_{i}$ are identical, which is denoted by $\tau_{i}$. In order to main the traffic conditions within each cordon area, an optimal toll pattern $\tau^{*}=\left(\tau_{i}^{*}, i=1,2, \ldots, I\right)^{\mathrm{T}}$ should be such that the total inbound flow to each cordon is restricted, i.e., the following flow capacity constraint should be fulfilled:

$$
\sum_{a \in \bar{A}_{i}} v_{a}\left(\boldsymbol{\tau}^{*}\right) \leq H_{i}, i=1,2, \ldots, I
$$

where $H_{i}$ is the predetermined threshold for total inbound flow to cordon $i$, which is constant. The mapping $v_{a}\left(\boldsymbol{\tau}^{*}\right)$ denotes the equilibrium link flow on link $a \in A$ in terms of toll pattern $\boldsymbol{\tau}^{*}=\left(\tau_{i}^{*}, i=1,2, \ldots, I\right)^{\mathrm{T}}$, which can be obtained by solving a traffic assignment problem based on the generalized link travel time function:

$$
T_{a}\left(\mathbf{v}, \boldsymbol{\tau}^{*}\right)=t_{a}(\mathbf{v})+\tau_{a}^{*} / \alpha, a \in A
$$


where toll charge on link $a \in A$ should be

$$
\tau_{a}^{*}=\sum_{i=1}^{I} \tau_{i} \lambda_{a}^{i}, a \in A
$$

Herein $\lambda_{a}^{i}=1$ if $a$ is an entry link to cordon $i$ and $\lambda_{a}^{i}=0$, otherwise. $\alpha$ here is the drivers' value-of-time (VOT). The framework for traffic assignment in this paper follows the Stochastic User Equilibrium (SUE) proposed by Daganzo and Sheffi (1977) in view of its better representativeness to practical conditions. The mathematical model and solution algorithm for the SUE problem based on the generalized link travel time function (5) are briefly introduced in the following sub-section.

\subsection{Asymmetric Stochastic User Equilibrium Problem}

Assumption 2. The network users are assumed to make their route choice based on the perceived path travel time:

$$
C_{w k}(\mathbf{v}, \boldsymbol{\tau})=\sum_{a \in A} T_{a}(\mathbf{v}, \boldsymbol{\tau}) \delta_{a k}^{w}+\zeta_{w k}, k \in R_{w}, w \in W
$$

where $\zeta_{w k}$ is the users' perception error on the path travel time

Therefore, with any given toll charge pattern $\tau=\left(\tau_{i} \geq 0, i=1,2, \ldots, I\right)^{\mathrm{T}}$, the network users' responses are analyzed by solving a SUE traffic assignment problem. The perception error in Eq. (7) is usually assumed to follow Gumbel distribution (Logit-based SUE) or Normal distribution (Probit-based SUE). Let $S_{w}(\mathbf{v}, \boldsymbol{\tau})$ be the expected value of the minimum perceived travel time among all the paths between OD pair $w \in W$, thus $S_{w}(\mathbf{v}, \boldsymbol{\tau})=E\left[\min _{k \in R_{w}}\left\{C_{w k}(\mathbf{v}, \boldsymbol{\tau})\right\}\right]$. In the literature, $S_{w}(\mathbf{v}, \boldsymbol{\tau})$ is also termed as satisfaction or satisfaction function (Sheffi, 1985).

The users are assumed to choose the path with minimal perceived travel time, and the route choice problem is then analyzed by discrete choice model (Daganzo and Sheffi, 1977). Let $p_{w k}(\mathbf{v}, \boldsymbol{\tau})$ be the probability that path $k$ is taken as the shortest one by the users between OD pair $w \in W$. Thus, for the SUE problem with asymmetric link travel 
time function addressed in this paper, it can be formulated by the following fixed-point model proposed by Daganzo (1983):

$$
v_{a}=\sum_{w \in W}\left[q_{w} \times P_{w a}(\mathbf{v}, \boldsymbol{\tau})\right], a \in A
$$

where $P_{w a}(\mathbf{v}, \boldsymbol{\tau})=\sum_{k \in R_{w}} p_{w k}(\mathbf{v}, \boldsymbol{\tau}) \delta_{a k}^{w}, w \in W$. Based on the feasible set of link flows defined by the flow conservation eqs. (1)-(3), the existence and uniqueness of solution to model (8) can be guaranteed (Cantarella, 1997).

The fixed point model (8) is usually solved by the Method of Successive Average (MSA) with predetermined step sizes (e.g., Daganzo, 1983; Cantarella, 1997). The MSA iteratively invokes the calculation of link-travel-time functions and the stochastic network loading map. The stochastic network loading map plays the same role as all-or-nothing assignment in solving Deterministic User Equilibrium (DUE) problem (Sheffi, 1985). Solution algorithms for performing stochastic network loading have been well investigated in the literature both for logit-based SUE (see, Dial, 1971; Bell, 1995) and probit-based SUE (e.g., Maher and Hughes, 1997; Meng and Liu, 2012b), which are not included here due to the space limit.

An extension of MSA method can be observed in Liu et al. (2009), where selfadaptive step sizes are used, which contributes to a much faster computational speed compared with the MSA.

\subsection{Mathematical Conditions for the Optimal Lagrangian Multipliers}

As mentioned in the Introduction section, the optimal toll charge pattern can be achieved by first solving a traffic assignment problem with side constraint (4), and then letting $\tau_{i} / \alpha, i \leq I$ equal the optimal Lagrangian multipliers associated to the side constraint of cordon $i$.

Assumption 3. Denoted by $\tau_{i}$, the toll charge on each link of cordon $i$ is identical. 
Suppose the optimal Lagrangian multipliers corresponding to the side constraints (4) are denoted by $\mathbf{u}^{*}=\left(u_{i}^{*}, i=1,2, \ldots, I\right)^{\mathrm{T}}$. The KKT conditions imply that the following conditions should be satisfied:

$$
\begin{gathered}
\sum_{a \in \bar{A}_{i}} v_{a}\left(\mathbf{u}^{*}\right) \leq H_{i}, i=1,2, \ldots, I \\
u_{i}^{*} \times\left(\sum_{a \in \bar{A}_{i}} v_{a}\left(\mathbf{u}^{*}\right)-H_{i}\right)=0, i=1,2, \ldots, I \\
u_{i}^{*} \geq 0, i=1,2, \ldots, I
\end{gathered}
$$

where eq. (10) is the complementary slackness condition and eq. (11) is the nonnegativity condition. The mapping $v_{a}(\mathbf{u}), a \in A$ denotes the SUE link flow in terms of the generalized link travel time functions:

$$
T_{a}(\mathbf{v}, \mathbf{u})=t_{a}(\mathbf{v})+u_{a}, a \in A
$$

where $u_{a}=\sum_{i=1}^{I} u_{i} \lambda_{a}^{i}, a \in A$ is the cumulative value of Lagrangian multipliers on link $a$. Then, the optimal toll charge pattern can be attained as $\tau_{i}^{*}=u_{i}^{*} \times \alpha, i=1,2, \ldots, I$. Eqs. (9)(11) are then taken as the mathematical definitions/conditions of the optimal Lagrangian multipliers $\mathbf{u}^{*}$ and optimal toll charge pattern $\tau^{*}$.

For the DUE with side constraints (including the link capacity constraints), a suitable formulation can be attained by directly adding the side constraints to the standard DUE models. For instance, adding side constraints to the Beckmann's transformation model (see Sheffi, 1985) gives us a successful model for the DUE with side constraints:

$$
\min Z_{1}=\sum_{a \in A} \int_{0}^{v_{a}} t_{a}(w) d w
$$

subject to

$$
\begin{gathered}
\sum_{k \in R_{w}} f_{w k}=q_{w} \\
f_{w k} \geq 0, k \in R_{w}, w \in W \\
\sum_{a \in \bar{A}_{i}} v_{a} \leq H_{i}, i=1,2, \ldots, I
\end{gathered}
$$


The KKT equations of nonlinear model (13)-(14) show that its optimal solution can still fulfill the DUE conditions in terms of generalized travel time functions (Larsson and Patriksson, 1999; Chen et al., 2011).

However, modeling for the SUE problem with side constraints is much more challenging due to the following phenomenon: when adding side constraints to the SUE model proposed by Sheffi and Powell (1982), we obtain the following model:

$$
\min Z_{2}=-\sum_{w \in W} q_{w} S_{w}(\mathbf{v})+\sum_{a \in A} v_{a} t_{a}\left(v_{a}\right)+\sum_{a \in A} \int_{0}^{v_{a}} t_{a}(x) d x
$$

subject to

$$
\sum_{a \in \bar{A}_{i}} v_{a} \leq H_{i}, i=1,2, \ldots, I
$$

Let $\mathbf{v}^{*}=\left(v_{a}^{*}, a \in A\right)^{\mathrm{T}}$ denote the optimal link flow solution to model (15)-(16). The KKT conditions imply that $v_{a}^{*}$ should fulfill the following condition:

$$
-\sum_{w \in W} q_{w} \sum_{k} p_{w k}\left(\mathbf{v}^{*}\right) \frac{d t_{a}}{d v_{a}^{*}} \delta_{a k}^{w}+v_{a}^{*} \frac{d t_{a}}{d v_{a}^{*}}-\sum_{i=1}^{I} \lambda_{a}^{i} u_{i}^{*}=0
$$

where $u_{i}^{*}$ is the optimal Lagrangian multiplier to side constraint $i$, and the incidence relationship factor $\lambda_{a}^{i}$ is defined in eq. (6). Clearly, $\mathbf{v}^{*}$ in eq. (17) cannot fulfill the equilibrium conditions in terms of generalized travel time functions, defined by eq. (8).

In contrast to the derivations for the standard SUE problem with no side constraints (see Page 317 in Sheffi, 1985), the phenomenon discussed above is caused because the third term in eq. (17) cannot be simply added to the path choice probability $p_{w k}\left(\mathbf{v}^{*}\right)$ in the first term. This phenomenon has largely limited the studies of SUE problems with side constraints. As mentioned in the Introduction section, the existing studies conducted by Meng et al. (2008) and Meng and Liu (2011) for SUE problem with side constraints assumed separable travel time function. Thus, their methodologies are not suitable for the asymmetric case addressed in this paper.

Since it is difficult to directly formulate the SUE problem with side constraints, we then try to only formulate the optimal Lagrangian multipliers defined by eq. (9)-(11), which are addressed in the following section. 


\section{VARIATIONAL INEQUALITY (VI) MODEL FOR THE OPTIMAL LAGRANGIAN MULTIPLIERS AND ITS SOLUTION ALGORITHMS}

When the feasible set of Lagrangian multipliers $\mathbf{u}=\left(u_{i}, i=1,2, \ldots, I\right)^{\mathrm{T}}$ is defined as the whole non-negative orthant, the conditions (9)-(11) are equivalent to the following VI model (He and Liao, 2002):

$$
\boldsymbol{\Phi}\left(\mathbf{u}^{*}\right)\left(\mathbf{u}-\mathbf{u}^{*}\right) \geq 0, \mathbf{u} \in \Omega
$$

where $\Omega=\left\{\mathbf{u} \mid u_{i} \geq 0, i=1,2, \ldots, I\right\}$ is the feasible set of $\mathbf{u}$ and the VI function $\boldsymbol{\Phi}(\mathbf{u})$ is defined as:

$$
\boldsymbol{\Phi}(\mathbf{u})=\left(\Phi_{i}(\mathbf{u}), i=1,2, \ldots, I\right)^{\mathbf{T}}=\left(H_{i}-\sum_{a \in A_{i}} v_{a}(\mathbf{u}), i=1,2, \ldots, I\right)^{\mathbf{T}}: \Omega \rightarrow \mathfrak{R}^{I}
$$

Therefore, by solving eq. (18), we can obtain the optimal Lagrangian multipliers that can fulfill conditions (9)-(11), and then get the optimal toll charge pattern. The theoretical convergence of many solution algorithms for eq. (18) requires the monotonicity of the VI function (19). Thus, the monotone property of VI function (19) is of considerable significance, which is highlighted in Proposition 1.

Proposition 1. The VI function (19) is monotone, namely

$$
\left(\boldsymbol{\Phi}\left(\mathbf{u}^{\prime}\right)-\boldsymbol{\Phi}\left(\mathbf{u}^{\prime \prime}\right)\right)^{\mathrm{T}}\left(\mathbf{u}^{\prime}-\mathbf{u}^{\prime \prime}\right) \geq 0, \forall \mathbf{u}^{\prime}, \mathbf{u}^{\prime \prime} \in \Omega
$$

Proof. For any two distinct non-negative Lagrangian-multiplier vectors $\mathbf{u}^{\prime}$ and $\mathbf{u}^{\prime \prime}$, we have the toll charge pattern $\boldsymbol{\tau}^{\prime}=\left(\alpha \times u_{i}^{\prime}, i=1,2, \ldots, I\right)^{\mathrm{T}}$ and $\boldsymbol{\tau}^{\prime \prime}=\left(\alpha \times u_{i}^{\prime \prime}, i=1,2, \ldots, I\right)^{\mathrm{T}}$. Let $\tau_{a}^{\prime}$ and $\tau_{a}^{\prime \prime}$ denote the corresponding toll charge on link $a \in A$, given by eq. (6). Firstly, eq. (20) is rewritten as: 


$$
\begin{aligned}
& \left(\boldsymbol{\Phi}\left(\mathbf{u}^{\prime}\right)-\boldsymbol{\Phi}\left(\mathbf{u}^{\prime \prime}\right)\right)^{\mathrm{T}}\left(\mathbf{u}^{\prime}-\mathbf{u}^{\prime \prime}\right)=\sum_{i=1}^{I}\left(H_{i}-\sum_{a \in \bar{A}_{i}} v_{a}\left(\mathbf{u}^{\prime}\right)-H_{i}+\sum_{a \in \bar{A}_{i}} v_{a}\left(\mathbf{u}^{\prime \prime}\right)\right)\left(u_{i}^{\prime}-u_{i}^{\prime \prime}\right) \\
& =-\sum_{i=1}^{I} \sum_{a \in \bar{A}_{i}}\left(v_{a}\left(\mathbf{u}^{\prime}\right)-v_{a}\left(\mathbf{u}^{\prime \prime}\right)\right)\left(u_{i}^{\prime}-u_{i}^{\prime \prime}\right)=-\sum_{a \in \bar{A}}\left(v_{a}\left(\mathbf{u}^{\prime}\right)-v_{a}\left(\mathbf{u}^{\prime \prime}\right)\right) \sum_{i=1}^{I}\left(u_{i}^{\prime}-u_{i}^{\prime \prime}\right) \delta_{a}^{i} \\
& =-\sum_{a \in \bar{A}}\left(v_{a}\left(\mathbf{u}^{\prime}\right)-v_{a}\left(\mathbf{u}^{\prime \prime}\right)\right)\left(\tau_{a}^{\prime}-\tau_{a}^{\prime \prime}\right) / \alpha
\end{aligned}
$$

Thus, eq. (20) equals to

$$
\sum_{a \in \bar{A}}\left(v_{a}\left(\mathbf{u}^{\prime}\right)-v_{a}\left(\mathbf{u}^{\prime \prime}\right)\right)\left(\tau_{a}^{\prime}-\tau_{a}^{\prime \prime}\right) \leq 0
$$

Since for those links with no side constraints, the toll charge is zero, thus it gives

$$
\sum_{a \in A \backslash \bar{A}}\left(v_{a}\left(\mathbf{u}^{\prime}\right)-v_{a}\left(\mathbf{u}^{\prime \prime}\right)\right)\left(\tau_{a}^{\prime}-\tau_{a}^{\prime \prime}\right) \equiv 0
$$

By summing up eqs. (22) and (23), we know that the monotone condition (20) is equivalent to

$$
\sum_{a \in A}\left(v_{a}\left(\mathbf{u}^{\prime}\right)-v_{a}\left(\mathbf{u}^{\prime \prime}\right)\right)\left(\tau_{a}^{\prime}-\tau_{a}^{\prime \prime}\right) \leq 0
$$

Eq. (24) is then proven as follows:

The satisfaction function $S_{w}(\mathbf{v}, \boldsymbol{\tau})$ for SUE problem is well recognized to be concave (e.g., Sheffi, 1985), and it implies that

$$
\begin{aligned}
& S_{w}\left(\mathbf{v}\left(\mathbf{u}^{\prime}\right), \boldsymbol{\tau}^{\prime}\right)-S_{w}\left(\mathbf{v}\left(\mathbf{u}^{\prime \prime}\right), \boldsymbol{\tau}^{\prime \prime}\right) \leq \sum_{r \in R_{w}}\left(p_{w r}\left(\mathbf{v}\left(\mathbf{u}^{\prime \prime}\right), \boldsymbol{\tau}^{\prime \prime}\right)\right)\left(\vec{c}_{w r}-\vec{c}_{w r}^{\prime \prime}\right), w \in W \\
& S_{w}\left(\mathbf{v}\left(\mathbf{u}^{\prime \prime}\right), \boldsymbol{\tau}^{\prime \prime}\right)-S_{w}\left(\mathbf{v}\left(\mathbf{u}^{\prime}\right), \boldsymbol{\tau}^{\prime}\right) \leq \sum_{r \in R_{w}}\left(p_{w r}\left(\mathbf{v}\left(\mathbf{u}^{\prime}\right), \boldsymbol{\tau}^{\prime}\right)\right)\left(\vec{c}_{w r}^{\prime \prime}-\vec{c}_{w r}^{\prime}\right), w \in W
\end{aligned}
$$

Adding up eqs. (25) and (26) yields that

$$
0 \geq \sum_{r \in R_{w}}\left(p_{w r}\left(\mathbf{v}\left(\mathbf{u}^{\prime}\right), \boldsymbol{\tau}^{\prime}\right)-p_{w r}\left(\mathbf{v}\left(\mathbf{u}^{\prime \prime}\right), \boldsymbol{\tau}^{\prime \prime}\right)\right)\left(\vec{c}_{w r}^{\prime}-\vec{c}_{w r}^{\prime \prime}\right), w \in W
$$

Multiplying by a non-negative value $q_{w}$, the two sides of eq. (27) then become

$$
0 \geq \sum_{r \in R_{w}}\left(f_{w r}\left(\mathbf{u}^{\prime}\right)-f_{w r}\left(\mathbf{u}^{\prime \prime}\right)\right)\left(\vec{c}_{w r}-\vec{c}_{w r}^{\prime \prime}\right), w \in W
$$


Accordingly, by summing up all the OD pairs, we have

$$
0 \geq \sum_{w \in W} \sum_{r \in R_{w}}\left(f_{w r}\left(\mathbf{u}^{\prime}\right)-f_{w r}\left(\mathbf{u}^{\prime \prime}\right)\right)\left(\vec{c}_{w r}-\vec{c}_{w r}^{\prime \prime}\right)
$$

Then, it gives

$$
0 \geq \sum_{w \in W} \sum_{r \in R_{w}}\left(f_{w r}\left(\mathbf{u}^{\prime}\right)-f_{w r}\left(\mathbf{u}^{\prime \prime}\right)\right)\left(\sum_{a \in A}\left(t_{a}\left(\mathbf{v}\left(\mathbf{u}^{\prime}\right)\right)+\tau_{a}^{\prime} / \alpha\right) \delta_{a r}^{w}-\sum_{a \in A}\left(t_{a}\left(\mathbf{v}\left(\mathbf{u}^{\prime \prime}\right)\right)+\tau_{a}^{\prime \prime} / \alpha\right) \delta_{a r}^{w}\right)(30)
$$

Thus, we have

$$
\sum_{a \in A}\left(v_{a}\left(\mathbf{u}^{\prime}\right)-v_{a}\left(\mathbf{u}^{\prime \prime}\right)\right)\left(\tau_{a}^{\prime}-\tau_{a}^{\prime \prime}\right) / \alpha \leq-\sum_{a \in A}\left(v_{a}\left(\mathbf{u}^{\prime}\right)-v_{a}\left(\mathbf{u}^{\prime \prime}\right)\right)\left(t_{a}\left(\mathbf{v}\left(\mathbf{u}^{\prime}\right)\right)-t_{a}\left(\mathbf{v}\left(\mathbf{u}^{\prime \prime}\right)\right)\right)
$$

Since the link travel time function vector $\mathbf{t}(\mathbf{v})$ is strictly monotone, the right hand side of eq. (31) is non-negative.

Thus,

$$
\sum_{a \in A}\left(v_{a}\left(\mathbf{u}^{\prime}\right)-v_{a}\left(\mathbf{u}^{\prime \prime}\right)\right)\left(\tau_{a}^{\prime}-\tau_{a}^{\prime \prime}\right) \leq 0
$$

which gives the proof.

The monotone property of the VI model (18) ensures the convergence of several existing solution algorithm methods solving a VI problem; for instance, the prediction and correction (PC) proposed by Korpelevich (1976). A theoretically more efficient method is the self-adaptive PC method proposed by He and Liao (2002), which is an extension of the PC method with advanced self-adaptive step sizes. The self-adaptive PC method maintains a linear convergent speed. In each iteration, the self-adaptive PC method would invoke the calculation of projection operations of any Lagrangianmultiplier vector. Since the feasible set of Lagrangian-multiplier vector is the whole nonnegative orthant, it is quite straightforward and effortless to calculate the projection operation; for instance, for any vector $\mathbf{u}^{\prime}$, its projection to the feasible set $\Omega$ of the Lagrangian multipliers, denoted by $P_{\Omega}\left[\mathbf{u}^{\prime}\right]$, equals to

$$
P_{\Omega}\left[\mathbf{u}^{\prime}\right]=\left(\max \left(0, u_{i}^{\prime}\right), i=1,2, \ldots, I\right)^{\mathrm{T}}
$$


Therefore, it is quite convenient and efficient to adopt the self-adaptive PC method to solve model (18). The detailed procedure of the self-adaptive PC method is not listed here. The interested readers can refer to He and Liao (2002).

It should be noted that in theoretical studies, most of the computational efforts of solving the self-adaptive PC method are allocated to getting the value of VI function $\boldsymbol{\Phi}\left(\mathbf{u}^{(n)}\right)$ (see Eq. (19)) for an updated value of Lagrangian multipliers $\mathbf{u}^{(n)}$ in the interaction $n$. The value of $v_{a}(\mathbf{u}), a \in \bar{A}_{i}, i=1,2, \ldots, I$ in Eq. (19) can be obtained via solving a standard SUE problem in terms of the generalized link travel time functions, Eq. (12). In practice, however, it is very straightforward to obtain the value of $v_{a}(\mathbf{u}), a \in \bar{A}_{i}, i=1,2, \ldots, I$, since it is actually the hourly traffic volume on the entry links, which can be easily and accurately gathered by the transaction data in each tool booth. This implies that when used in practice, this method for adjusting the tolls can obviate the data of OD demand, link travel time functions, as well as drivers' value-of-time, etc.

\section{CONCLUSIONS}

This paper addressed the determination of optimal toll pattern for cordon-based pricing, with the objective of restricting the total inbound flow to the cordon to a predetermined threshold. The toll charge on each entry of one cordon is decided to be identical, for the ease of distributing the toll fare information to the drivers and also for the convenience of drivers to recognize and memorize the toll fares. It should be noted that the toll charges on entries of different cordons are allowed to be different.

The challenges of modelling SUE problems with side constraints were first examined. A variational inequality (VI) model was then proposed for the SUE problem with side constraints, and it was shown that the optimal toll pattern equals the optimal Lagrangian multiplier associated with the side constraints. The monotone property of proposed VI model was rigorously proven, and a self-adaptive prediction and correction (PC) algorithm is suggested for solving the VI model. It should be pointed out that when adopted in practice, the solution method for adjusting the toll fares only needs the data of 
traffic counts on entry links, avoiding gathering the data of OD demand, link travel time functions, and drivers' value-of-time.

The optimal toll charge addressed in this paper is obtained by solving a side constrained traffic assignment problem. Compared with the first-best pricing, the total social benefit in this case could be compromised. This phenomenon implies that if the land transport authorities only focus on the traffic conditions in the cordon-area, traffic congestions in other network segments might be deteriorated. To this end, some further investigations are necessary on properly defining the threshold values to the total inflow of each cordon, with the objective of maximizing the total social benefit of the entire transport network.

Future efforts are also needed to investigate the toll determination problem for cordon-based pricing in a dynamic environment, where a time-differentiated toll pattern is levied. Since some of the current congestion pricing practices have been upgraded to be time-differentiated (e.g., the Electronic Road Pricing in Singapore and the congestion pricing trial in Stockholm), the dynamic issues of cordon-based pricing are of considerable practical significance. Moreover, some other practical issues should be further considered for the formulation to extend the work proposed in this paper; for instance, the demand elasticity, randomness of drivers' value-of-time, multi-vehicle types, vehicle occupancy (Yan et al., 2002), etc.

\section{REFERENCES}

Bell, M.G.H., 1995. Alternatives to Dial's logit assignment algorithm. Transportation Research Part B, 29, 287-295.

Bertsimas, D., and Tsitsiklis, J.N., 1997. Introduction to Linear Optimization. Athena Scientific, Belmont, Massachusetts.

Cantarella, G.E., 1997. A general fixed-point approach to multimode multi-user equilibrium assignment with elastic demand, Transportation Science, 31(2), 107128.

Chen, A., Zhou, Z., and Ryu, S., 2011. Modeling physical and environmental side constraints in traffic equilibrium problem. International Journal of Sustainable Transportation, 5(3), 172-197.

Daganzo, C.F., 1983. Stochastic network equilibrium with multiple vehicle types and asymmetric, indefinite link cost Jacobians, Transportation Science, 17, 282-300

Daganzo, C.F. and Sheffi, Y., 1977. On stochastic models of traffic assignment. Transportation Science, 11(3), 253-274. 
de Palma, A., Lindsey, R., 2011. Traffic congestion pricing methodologies and technologies. Transportation Research Part C, 19(6), 1377-1399.

Dial, R.B., 1971. A probabilistic multipath traffic assignment algorithm which obviates path enumeration. Transportation Research, 5, 83-111

Foo, T.S., 2000. An advanced demand management instrument in urban transport: electronic road pricing in Singapore. Cities, 17, 33-45.

He, B.S. and Liao L.Z., 2002. Improvements of some projection methods for monotone nonlinear variational inequalities. Journal of Optimization Theory and Applications, 112(1), 111-128.

Korpelevich, G.M., 1976. The extragradient method for finding saddle points and other problems. Ekonomika Matematicheskie Metody, 12, 747-756.

Larsson, T. and Patriksson, M., 1995. An augmented Lagrangean dual algorithm for link capacity side constrained traffic assignment problems. Transportation Research Part B, 29(6), 433-455.

Larsson, T. and Patriksson, M., 1999. Side constrained traffic equilibrium models analysis, computation and applications. Transportation Research Part B, 33(4), 233-264.

Lawphongpanich S., Hearn, D.W., Smith, M. J., 2006. Mathematical and Computational Models for Congestion Charging, Springer.

Liu, H.X., He, X. and He, B., 2009. Method of successive weighted averages (MSWA) and self-regulated averaging schemes for solving Stochastic User Equilibrium problem. Networks and Spatial Economics, 9, 485-503.

Liu, Z., 2011. Probit-based Stochastic User Equilibrium Problems and their Applications in Congestion Pricing. Ph.D Thesis, Department of Civil and Environmental Engineering, National University of Singapore.

Liu, Z., and Meng, Q., 2011. Distributed computing approaches for large-scale probitbased Stochastic User Equilibrium problems. Journal of Advanced Transportation, DOI: $10.1002 /$ atr. 177.

Liu, Z. and Meng, Q., 2012. Modelling Transit-based Park-and-Ride Services on a Multimodal Network with Congestion Pricing Schemes, International Journal of Systems Science, DOI:10.1080/00207721.2012.743617.

Liu, Z. and Meng, Q. and Wang, S., 2013. Speed-based Toll Design for Cordon-Based Congestion Pricing Scheme, Transportation Research Part C, 31, 83-98.

Maher M.J. and Hughes P.C., 1997. A probit-based stochastic user equilibrium assignment model. Transportation Research Part B, 31(4), 341-355.

Meng, Q., Lam, W. H.K., and Yang, L., 2008. General Stochastic User Equilibrium Traffic Assignment Problem with Link Capacity Constraints. Journal of Advanced Transportation, 42, 429-465.

Meng, Q. and Liu, Z., 2011. Trial-and-error method for congestion pricing scheme under side-constrained probit-based stochastic user equilibrium conditions. Transportation, 38, 819-843.

Meng, Q., and Liu, Z., 2012a. Impact analysis of cordon-based congestion pricing scheme on mode-split of bimodal transportation network. Transportation Research Part C, 21, 134-147. 
Meng, Q., and Liu, Z., 2012b. Mathematical models and computational algorithms for probit-based asymmetric stochastic user equilibrium problem with elastic demand. Transportmetrica, 8(4), 261-290.

Meng, Q., Liu, Z. and Wang, S., 2012. Optimal Distance-based Toll Design For Cordonbased Congestion Pricing Scheme with Continuously Distributed Value-of-time, Transportation Research Part E, 48(5), 937-957.

Meng, Q. and Liu, Z. and Wang S, 2013. Asymmetric Stochastic User Equilibrium Problem with Link Capacity Constraints and Elastic Demand, Transportmetrica. DOI:10.1080/23249935.2013.765929

Mun, S., Konishi, K. and Yoshikawa, K., 2003. Optimal cordon pricing. Journal of Urban Economics, 54, 21-38.

Nagurney, A., 1993. Network economics: a variational inequality approach. Kluwer Academic publishers, Dordrecht, Boston, London.

Nagurney A., and Ramanujam, P., 1998. A multimodal traffic network equilibrium model with emission pollution permits: compliance VS noncompliance. Transportation Research Part D, 3(5), 349-374.

Nie. Y., Zhang, H.M. and Lee, D.H., 2004. Models and algorithms for the traffic assignment problem with link capacity constraints. Transportation Research Part $B, 38(4), 285-312$.

Pigou, A.C., 1920. The Economics of Welfare. MacMillan, London.

Shahpar, A.H., Aashtiani, H.Z. and Babazadeh, A., 2008. Dynamic penalty function method for the side constrained traffic assignment problem. Applied Mathematics and Computation, 206, 332-345.

Sheffi, Y., 1985. Urban Transportation Networks: Equilibrium analysis with Mathematical Programming Models. Prentice-Hall, INC, Englewood Cliffs, New Jersey.

Sheffi, Y. and Powell, W.B., 1982. An algorithm for the equilibrium assignment problem with random link times. Networks, 12(2), 191-207.

Verhoef, E.T., 2002. Second-best congestion pricing in general networks: heuristic algorithms for finding second-best optimal toll levels and toll points. Transportation Research Part B, 36, 707-729.

Yan, J., Small, K.A., and Sullivan, E.C., 2002. Choice models of route, occupancy, and time of day with value-priced tolls, Transportation Research Record, 1812, 69-77.

Yang, H. and Huang, H. J., 2005. Mathematical and Economic Theory of Road Pricing. Elsevier Ltd.

Yang, H., Zhang, X., 2002. The multi-class network toll design problem with social and spatial equity constraints. Journal of Transportation Engineering, ASCE 128, 420-428.

Zhang X.N. and Yang, H., 2004. The optimal cordon-based network congestion pricing problem. Transportation Research Part B, 38, 517-537. 